\title{
Correction to: Characteristics of single ocular motor nerve palsy associated with anti-GQ1b antibody
}

\author{
Kwang-Dong Choi ${ }^{1}$. Seo Young Choi ${ }^{1}$. Jae-Hwan $\mathrm{Choi}^{2} \cdot$ Seong-Hee Kim ${ }^{3} \cdot$ Seung-Han Lee ${ }^{4}$. Seong-Hae Jeong ${ }^{5}$. \\ Hyo-Jung Kim ${ }^{6,7}$. Jeong-Yoon $\mathrm{Choi}^{7,8} \cdot \mathrm{Ji}^{\mathrm{i}-S o o} \mathrm{Kim}^{7,8}$ (D)
}

Published online: 4 January 2019

○) Springer-Verlag GmbH Germany, part of Springer Nature 2019

\section{Correction to: Journal of Neurology}

https://doi.org/10.1007/s00415-018-9161-8

The original version of this article unfortunately contained a mistake.

Names of co-authors were wrong. Names should be as follows:

Seong Hi Kim to Seong-Hee Kim

Seong-Han Lee to Seung-Han Lee

In the abstract, sixth sentence should read as:

None of the 30 patients with OMNP with identifiable causes showed positive serum anti-GQ1b antibody.

The original article can be found online at https://doi.org/10.1007/ s00415-018-9161-8.

\section{Ji-Soo Kim}

jisookim@snu.ac.kr

1 Department of Neurology, Biomedical Research Institute, Pusan National University Hospital, Pusan National University School of Medicine, Busan, South Korea

2 Department of Neurology, Biomedical Research Institute, Pusan National University Yangsan Hospital, Pusan National University School of Medicine, Busan, South Korea

3 Department of Neurology, Kyungpook National University School of Medicine, Daegu, South Korea

4 Department of Neurology, Chonnam National University Medical School, Chonnam National University Hospital, Gwangju, South Korea
5 Department of Neurology, Chungnam National University School of Medicine, Daejeon, South Korea

6 Department of Neurology, Dizziness Center, Seoul National University Bundang Hospital, Seongnam, South Korea

7 Research Administration Team, Seoul National University Bundang Hospital, Seongnam, South Korea

8 Department of Neurology, Seoul National University College of Medicine, Seoul National University Bundang Hospital, 300 Gumi-dong, Bundang-gu, Seongnam, Gyeonggi 463-707, South Korea 